\title{
Effect of ball-milling on preparation of composites of betulin and betulin diacetate with polyethylene glycol
}

\author{
T.P. Shakhtshneider ${ }^{1,2}$, M.A. Mikhailenko ${ }^{2}$, V.A. Drebushchak ${ }^{2,3}$, T.N. Drebushchak ${ }^{1,2}$, \\ S.A. Kuznetsova ${ }^{4,5}$ \\ ${ }^{1}$ Institute of Solid State Chemistry and Mechanochemistry SB RAS, Kutateladze, 18, Novosibirsk, \\ 630128 Russia \\ ${ }^{2}$ Novosibirsk State University, Pirogov str., 2, Novosibirsk, 630090 Russia \\ ${ }^{3}$ Sobolev Institute of Geology and Mineralogy SB RAS, Lavrentiev ave, 3, Novosibirsk, 630090 \\ Russia \\ ${ }^{4}$ Institute of Chemistry and Chemical Technology SB RAS, Federal Research Center \\ “Krasnoyarsk Science Center SB RAS”, Akademgorodok, 50/24, Krasnoyarsk, Russia \\ ${ }^{5}$ Siberian Federal University, Svobodny pr., 79/10, Krasnoyarsk, 660041, Russia
}

\begin{abstract}
The composites of betulin and betulin diacetate with polyethylene glycol (PEG) were prepared by ball-milling. In the case of betulin - PEG mixtures, the almost complete amorphization was observed under ball-milling. In contrast, for ball-milled mixtures of BDA with PEG, there were no significant changes in intensity of the lines at the powder X-ray diffraction patterns. The dissolution of BDA in the PEG melts formed at the contacts of particles under collisions with milling bodies leading to recrystallization of the drug was suggested. Hot stage optical microscopy study confirmed the possibility of dissolving of BDA crystals in PEG melt.
\end{abstract}

Keywords Betulin, Betulin diacetate, PEG, Ball-milling, DSC

\section{Introduction}

It is well known that PEG is a very promising solubilizing agent and is often used for preparation of solid dispersions, or composites, of the drugs $[1,2]$. Due to low melting temperature of PEG, the melting technique was often used for solid dispersion preparation [2, 3]. Mechanochemical methods also can be used to obtain the composites of drugs with PEG [4, 5]. The mechanism of composite formation during ball-milling is not yet clear. In particular, the interaction of the components can be facilitated by melting of solids at the contacts of particles under collisions or ball impacts. This mechanism can be expected in the case of the mixtures of the drugs with PEG due to its low melting point. 
Betulin and the related compounds found in the outer bark of the birch tree have attached an increasing interest due to their pharmacological activity. Betulin and its esters exhibit very poor solubility; therefore approaches are required to increase the rate of dissolution and solubility such as preparation of composites with polymers [6, 7]. In the previous papers, we reported on preparation of composites of betulin and betulin diacetate with water-soluble polymers, polyvinylpyrrolidone and polyethylene glycol, by ball-milling [8, 9]. They exhibited higher solubility of betulin and an improvement of its gastroprotective properties. The betulinPEG mechanocomposite demonstrated also an excellent effect on acute renal failure in rats compared with raw betulin. In contrast, the composites of betulin diacetate with PEG did not reveal the increased solubility of the drug compared to the initial substance.

The knowledge of mechanism of formation of composites of betulin and the related compounds with carriers such as PEG may contribute to evaluation of their pharmacological properties. Application of thermal analysis methods is useful for better understanding the behavior of compounds or mixtures of different components under ball-milling accompanying by heating the samples. The purpose of this work was to study the behavior of the composites of betulin and betulin diacetate in the mixtures with PEG under heating by thermal analysis methods.

\section{Experimental}

Materials

Betulin and betulin diacetate (BDA) were isolated from birch bark at the Institute of Chemistry and Chemical Technology SB RAS (Krasnoyarsk, Russia) according to developed methods [10, 11]. Betulin was purified by recrystallization from ethanol following by decomposition of the obtained betulin-ethanol solvate [12] at $180^{\circ} \mathrm{C}$ during $30 \mathrm{~min}$. The content of lupeol in the sample, according to HPLC data, did not exceed $1.5-2.5 \mathrm{wt} \%$. Betulin diacetate contained $\sim 3 \mathrm{wt} \%$ of two different betulin monoacetates and used without purification. PEG $\left(\mathrm{M}_{\mathrm{r}}=4000\right)$ (SigmaAldrich) was used without previous purification and drying.

\section{Ball-milling experiments}

The mixtures of betulin or BDA with PEG were ball-milled in a SPEX-8000 mixer mill (CertiPrep Inc., USA). Volume of steel vials $60 \mathrm{~mL}$, diameter of the balls $6 \mathrm{~mm}$, ratio of mass of sample to mass of balls 1:30, acceleration of a ball - 8-10 g. Time of treatment was 15 and 30 min. Ratio of the components, betulin (BDA) - polymer, was $1: 3$ or $1: 10(\mathrm{w} / \mathrm{w})$. For comparison, the physical mixtures were prepared by simple mixing of the components in the same ratios. 
High-performance liquid chromatography

The HPLC analysis was performed on a Milikhrom A-02 (ZAO EcoNova, Russia) with a ProntoSIL 120-5C18 AQ analytical column (2.0 mm $\times 75 \mathrm{~mm}$ i.d., $5 \mu \mathrm{m}$ particle size) (Merck, Darmstadt, Germany). The mobile phase was composed of acetonitrile (A) and phosphate buffer (B). Phosphate buffer was prepared from chromatography buffer pH 6.86 (Merck, Germany). The gradient was as follows: $0-3 \mathrm{~min}, 80-100 \%$ (solvent A), flow rate of $0.2 \mathrm{~mL} / \mathrm{min}$; 4-12.5 min, 100-100\% (solvent A), flow rate of $0.2 \mathrm{~mL} / \mathrm{min}$. The temperature of the column was maintained at $35^{\circ} \mathrm{C}$, and the effluent was monitored at 200, 210 and $220 \mathrm{~nm}$.

DSC

Thermoanalytical measurements were carried out using DSC-204 (Netzsch). Standard aluminum crucibles were not sealed, only covered with a lid; heating rate of $6 \mathrm{~K} \mathrm{~min}^{-1}$; gas (argon) flow of $20 \mathrm{~mL} \mathrm{~min}^{-1}$; sample mass of 5-10 mg.

X-ray diffraction analysis

X-ray powder diffraction patterns were measured using D8 DISCOVER diffractometer with GADDS two-coordinate detector (Bruker), $\mathrm{CuK}_{\alpha}$-radiation.

Hot-stage polarizing light microscopy study

Hot-stage polarizing light microscopy (HSM) study was performed using Polam microscope with photocamera. Hand-made hot table was used for sample heating. The temperature was monitored by means of a contact thermometer with a copper-constantan thermocouple. The rate of temperature changing was $5-7 \mathrm{C} / \mathrm{min}$.

\section{Results and discussion}

The HPLC data showed that there was no degradation of the substances under ball-milling at these conditions.

In the powder X-ray diffraction patterns, an amorphous state of betulin and BDA was detected when they were ball-milled alone (Figs. 1 and 2). In the case of BDA, there were two exothermic peaks at 80 and $125^{\circ} \mathrm{C}$ at the DSC curves which can be attributed to recrystallization of the substance and a broad endothermic peak corresponding to melting (Fig. 3). For the sample heated at the temperatures of exothermic peaks, the new peaks appeared suggesting crystallization of a new BDA polymorph under heating ball-milled BDA (Fig. 4(добавить)). Thus, two methods, X-ray diffraction analysis and DSC, confirmed that a new polymorph of 
BDA was crystallized from the BDA amorphous state obtained by ball-milling. It is an interesting fact because usually, amorphous forms prepared by grinding possess characteristics of the original polymorph, corresponding to 'polymorphic memory', and should crystallize to the original polymorph [13].

In the case of ball-milled betulin - PEG mixtures, the broadening peaks and decreasing their intensity was observed (Fig. 1) evidencing disordering of betulin crystal structure and its partial amorphization. In contrast, for ball-milled mixtures of BDA with PEG, there were no significant changes in intensity of the lines (Fig. 2). This can be seen more precisely at the Fig. 5 (этот рисунок перенести в Insert) where the intensity of the peaks of the mixtures was 4 times increased to see the peaks of the drugs.

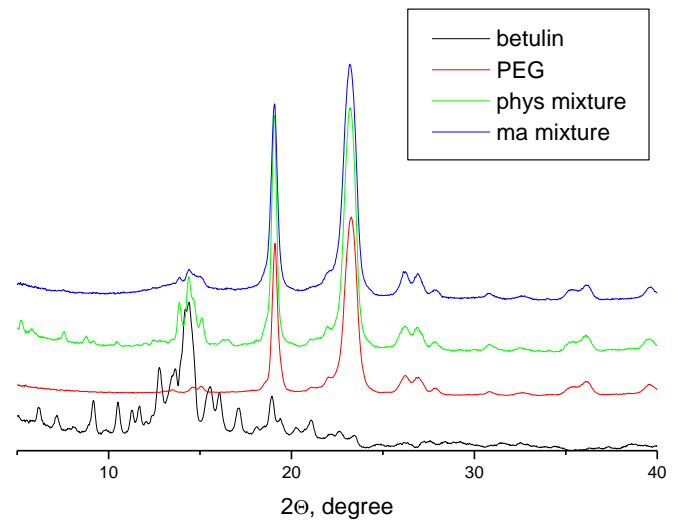

Fig. 1. Powder X-ray diffraction patterns of initial betulin and PEG, ball-milled betulin (добавить), 1:3 betulin-PEG physical and ball-milled mixtures.

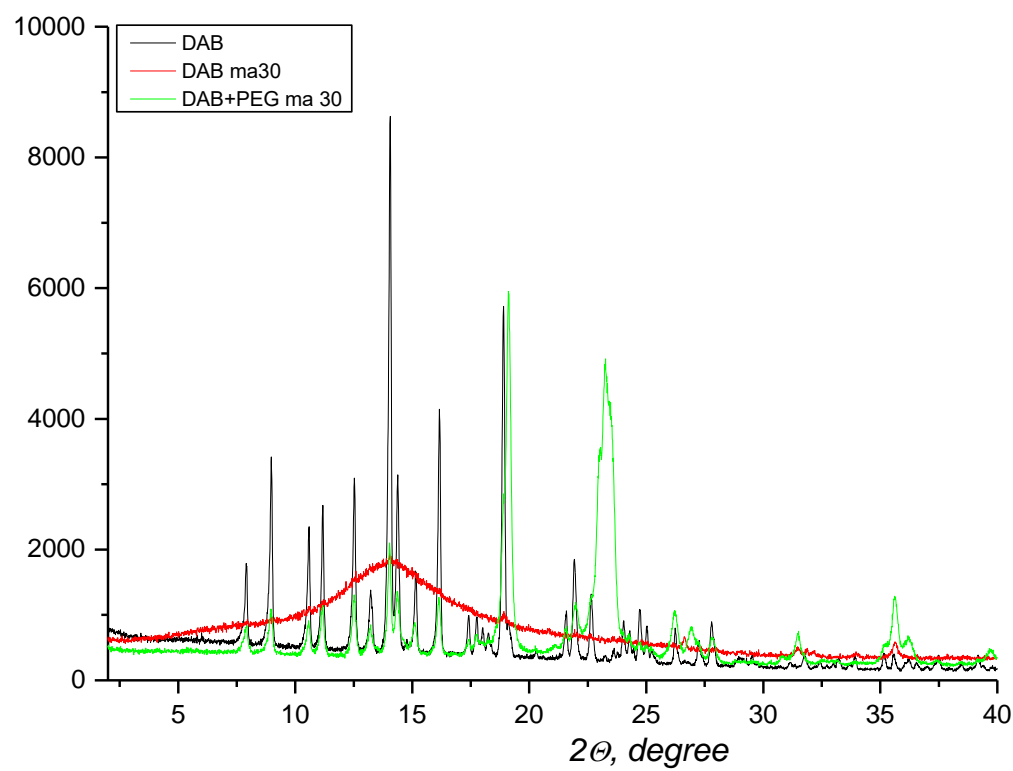

Fig. 2. Powder X-ray diffraction patterns of initial BDA, ball-milled BDA, 1:3 BDA-PEG physical (добавить) and ball-milled mixtures. 

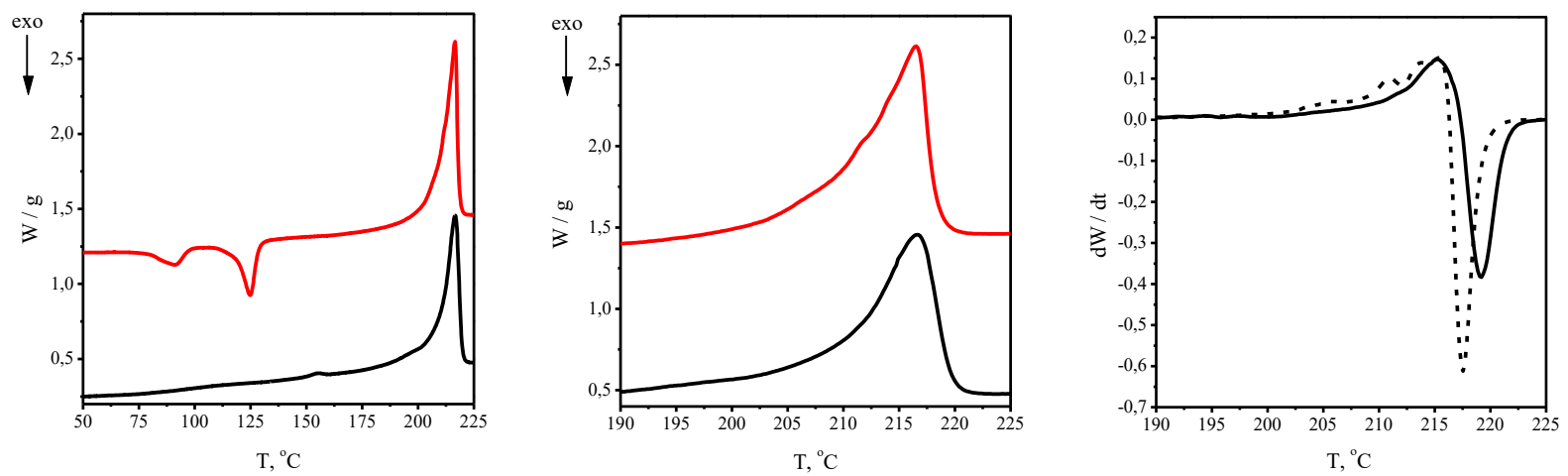

Fig. 3. DSC curves of initial and ball-milled BDA. И еще можно добавить такой же рисунок про бетулин
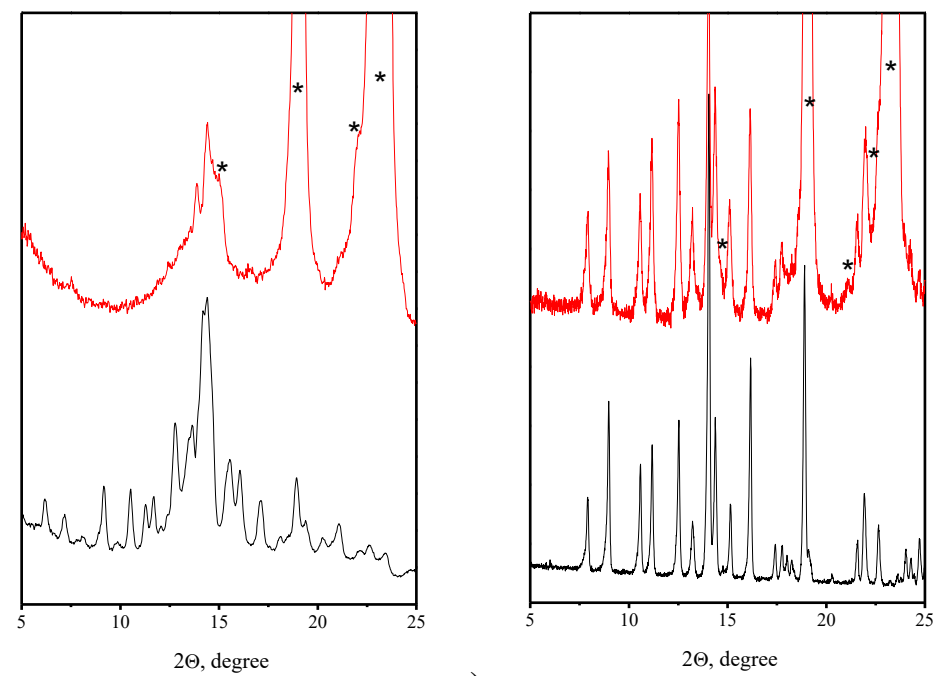

a)

b)

Fig. 5. Powder X-ray diffraction patterns of initial substance (down) and its mixtures witch PEG ( $1: 3, w / w)$ after grinding (up): a) betulin, b) betulin diacetate. The patterns of the mixtures are 4 times increased. The peaks of PEG are marked by asterisks.

This effect can be explained by dissolution of BDA in the melt of PEG followed by its crystallization from the melt. The fact that the peaks of original polymorph was observed at powder X-ray diffraction pattern of mechanocomposite confirmed that under ball milling crystallization proceeded not from amorphous state but probably from the melt.

It is known that the drugs can dissolve in PEG at heating [14-17]. It was suggested in [14] that indomethacin dissolved in the melt of PEG under mechanical activation not forming, according to IR spectroscopy data, intermolecular bonds with the polymer. Due to low temperature of melting of PEG $\left(61-63{ }^{\circ} \mathrm{C}\right)$, the formation of its melt at the contacts of particles under collisions and balls hitting is possible under ball-milling, therefore the recrystallization of BDA proceeded without its substantial amorphization. The presence of amorphous state in the 
ball-milled mixtures of betulin with PEG can be explained by suggestion that at these temperatures, betulin practically did not dissolve in PEG.

Fig. 6 shows the DSC thermograms of betulin - PEG and BDA - PEG physical mixtures. After PEG melting, some endothermic peaks were observed which were different at the DSC curves for betulin - PEG and BDA - PEG mixtures. In particular, for betulin - PEG mixture, the temperature of endothermic effect after PEG melting is higher than for BDA - PEG mixture suggesting that betulin can dissolve in the PEG melt at rather high temperature.
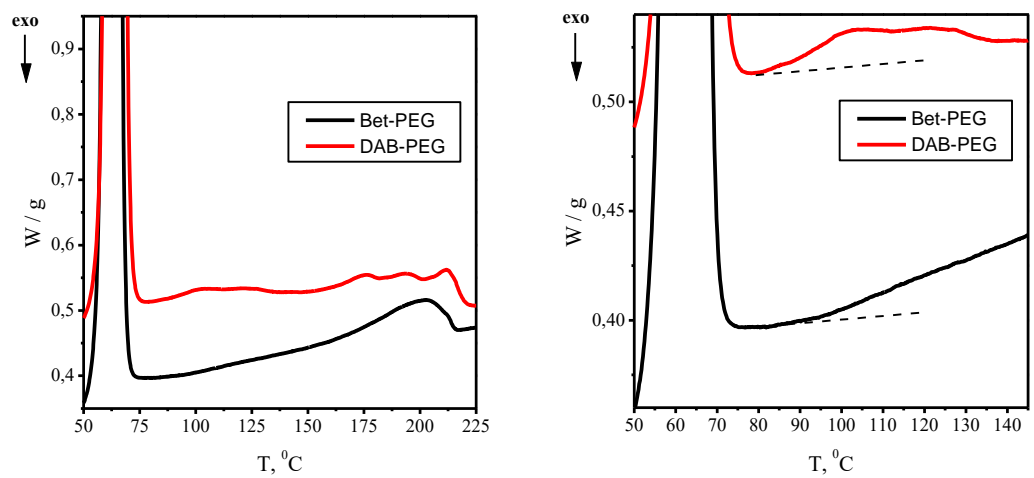

Fig. 6. DSC curves of betulin - PEG and BDA - PEG mixtures.

HSM study showed decreasing the size of BDA drug particles immediately after PEG melting that proved the dissolution of the drug in the melt of PEG on heating. Under cooling the melt, the formation of new crystals of rod morphology was observed.

The lack of amorphization of BDA under ball-milling with PEG may be due to local PEG melting at the contacts of the particles under collision with grinding bodies. Since simultaneously with the release of heat in the area of "strike", heat energy release by grinding bodies is also occurs, we can assume that the time of existence of a molten state is very small and, consequently, significant overheating relative to the melting point is likely not observed. Thus, under ball-milling BDA - PEG mixture, simultaneously with disordering the BDA crystal structure the defects disappearing proceeded due to dissolution of BDA in the melt and subsequent crystallization.

In the mixtures of betulin with PEG, due to higher temperature of betulin dissolution in the melt, the "annealing" of defects was less pronounced resulting in disordering its crystal structure under ball-milling.

\section{Conclusions}

It has been shown by X-ray analysis that in the case of betulin - PEG mixtures, the partial amorphization was observed under ball-milling. In contrast, for ball-milled mixtures of BDA 
with PEG, there were no significant changes in intensity of the lines at the powder X-ray diffraction patterns. The dissolution of BDA in the PEG melts formed at the contacts of particles under collisions with milling bodies was suggested leading to recrystallization of the drug. Hot stage optical microscopy study confirmed the possibility of dissolving crystals of BDA in PEG melt. The DSC method showed that betulin and BDA behaved differently in the mixtures with PEG under heating. Dissolution of betulin in PEG proceeded at higher temperatures than dissolution of BDA resulting in disordering its crystal structure under ball-milling.

\section{References}

1. Leuner C, Dressman J. Improving drug solubility for oral delivery using solid dispersions. Eur. J. Pharm. Biopharm. 2000;50:47-60.

2. Ladan Akbarpour Nikghalb, Gurinder Singh, Gaurav Singh, Kimia Fazaeli Kahkeshan. Solid dispersion: Methods and polymers to increase the solubility of poorly soluble drugs. Journal of Applied Pharmaceutical Science. 2012;2:170-175.

3. Mohan A, Madhavi M, Jyosthna P. Preparation, in vitro and in vivo characterization of solid dispersions of Oxcarbazepine using melting technique. Pharma Innovation. 2015;3:99-103.

4. Shakhtshneider TP, Boldyrev VV, Vasilchenko MA, Politov AA. The mechanochemical preparation of solid disperse systems of ibuprofen-polyethylene glycol. Int. J. Pharmaceutics. 1996;130:25-32.

5. Colombo I, Grassi G, Grassi M. Drug mechanochemical activation. Journal of Pharmaceutical Sciences. 1998;98:3961-3986.

6. Alakurtti S, Mäkelä T, Koskimies S, Yli-Kauhaluoma J. Pharmacological properties of the ubiquitous natural product betulin. European Journal of Pharmaceutical Sciences. 2006;29:113.

7. Tolstikova TG, Sorokina IV, Tolstikov GA, Tolstikov AG, Flekhter OB. Biological activity and pharmacological prospects of lupane terpenoids: I. Natural lupane derivatives. Russian Journal of Bioorganic Chemistry. 2006;32:37-49.

8. Mikhailenko MA, Shakhtshneider TP, Brezgunova ME, Kuznetsova SA, Boldyrev VV. The composition of betulin with biocompatible polymers and the method of its preparation. Russian Federation patent No. 401118. 2010.

9. Mikhailenko MA, Shakhtshneider TP, Drebushchak VA, Kuznetsova SA, Skvortsova GP, Boldyrev VV. Influence of mechanical treatment on the properties of betulin, betulin diacetate, and their mixture with water-soluble polymers. Chemistry of Natural Compounds. 2011;47:229-233. 
10. Kuznetsova SA, Kuznetsov BN, Mikhailov AG, Levdanskii VA. The method of obtaining of betulin. Russian Federation patent No. 2264411. Бюллетень изобретений, № 32, 473 (2005).

11. Kuznetsova SA, Kuznetsov BN, Red'kina ES, Sokolenko VA, Skvortsova GP. The method of obtaining of betulin diacetate. Russian Federation patent No. 2324700. Бюллетень изобретений, № 14, 689 (2008).

12. Drebushchak TN, Mikhailenko MA, Brezgunova ME, Shakhtshneider TP, Kuznetsova SA. Crystal structure of betulin ethanol solvate. Journal of Structural Chemistry. 2010;51:798801.

13. Sheth AR, Lubach JW, Munson EJ, Muller FX, Grant DJW. Mechanochromism of piroxicam accompanied by intermolecular proton transfer probed by spectroscopic methods and solidphase changes. J. Am. Chem. Soc. 2005;127:6641- 6651.

14. Fini A, Rodriguez L, Cavallarib C. Ultrasound-compacted and spray-congealed indomethacin. Polyethylene glycol systems. Int. J. Pharm. 2002;247:11-22.

15. Bikiaris D, Papageorgiou GZ, Stergiou A, Pavlidou E, Karavas E, Kanaze F, Georgarakis M. Physicochemical studies on solid dispersions of poorly water-soluble drugs: Evaluation of capabilities and limitations of thermal analysis techniques. Thermochimica Acta. 2005;439:58-67

16. Fini A, Moyano JR, Juan M, Perez-Martinez JI, Rabasco AM. Diclofenac salts, II. Solid dispersions in PEG 6000 and Gelucire 50/13. European Journal of Pharmaceutics and Biopharmaceutics. 2005;60:99-111.

17. Kumar SGV, Mishra DN. Preparation, characterization and in vitro dissolution studies of solid dispersion of meloxicam with PEG 6000. Yakugaku Zasshi - J. Pharm. Soc. Japan. 2006;126:657-664. 